\title{
Derivation of the Drude conductivity from quantum kinetic equations
}

AUTHOR(S):

Kitamura, Hikaru

CITATION:

Kitamura, Hikaru. Derivation of the Drude conductivity from quantum kinetic equations. European Journal of Physics 2015, 36: 065010.

\section{ISSUE DATE:}

2015-11-01

URL:

http://hdl.handle.net/2433/218783

\section{RIGHT:}

This is an author-created, un-copyedited version of an article accepted for publication in 'European Journal of Physics'. The publisher is not responsible for any errors or omissions in this version of the manuscript or any version derived from it. The Version of Record is available online at https://doi.org/10.1088/0143-0807/36/6/065010.; この論文は出版社版で ありません。引用の際には出版社版をご確認ご利用ください。;This is not the published version. Please cite only the published version. 


\title{
Derivation of the Drude conductivity from quantum kinetic equations
}

\author{
Hikaru Kitamura \\ Department of Physics, Kyoto University, Sakyo-ku, Kyoto 606-8502, Japan \\ E-mail: kitamura@scphys.kyoto-u.ac.jp
}

\begin{abstract}
The Drude formula of ac (frequency-dependent) electric conductivity has been established as a simple and practically useful model to understand the electromagnetic response of simple freeelectron-like metals. In most textbooks of solid-state physics, the Drude formula is derived from either a classical equation of motion or the semiclassical Boltzmann transport equation. On the other hand, quantum-mechanical derivation of the Drude conductivity, which requires an appropriate treatment of phonon-assisted intraband transitions with small momentum transfer, has not been well documented except for zero- or high-frequency case. Here, a lucid derivation of the Drude conductivity that covers the entire frequency range is presented by means of quantum kinetic equations in the density-matrix formalism. The derivation is straightforward so that advanced undergraduate students or early-year graduate students will be able to gain insight into the link between the microscopic Schrödinger equation and macroscopic transport.
\end{abstract}




\section{INTRODUCTION}

Electron conduction in a metal is an elementary subject treated in most textbooks of solid-state physics [1-3]. When a time-varying electric field $\mathbf{E}(t)=\mathbf{E}_{0} \exp (-i \omega t)$ is applied to a metal, each electron is accelerated by the field and then scattered by phonons, impurities, and so on. If the field is weak enough, the current density induced in the system may be expressed in the form $\mathbf{J}(t)=\mathbf{J}_{0} \exp (-i \omega t)$. The complex electric conductivity $\tilde{\sigma}(\omega)$, defined through the linear relationship $\mathbf{J}_{0}=\tilde{\sigma}(\omega) \mathbf{E}_{0}$, characterizes the response of the material to an electromagnetic wave of frequency $\omega$ [3]; it reduces to the dc electric conductivity at $\omega=0$.

The free-electron theory pioneered by Drude and Sommerfeld achieved a considerable success in the theory of electric conductivity [1]. The Drude formula for $\tilde{\sigma}(\omega)$ reads [1-3]

$$
\tilde{\sigma}(\omega)=\frac{n_{\mathrm{e}} e^{2} \tau}{m_{*}} \frac{1}{1-i \omega \tau},
$$

where $e$ stands for the elemental charge, $n_{\mathrm{e}}$ denotes the electron number density, $m_{*}$ refers to the effective mass of an electron which generally differs from the bare electron mass $m_{\mathrm{e}}$, and $\tau$ represents the mean time interval between two successive collisions that an electron encounters, called the relaxation time. In a crystalline metal, $m_{*}$ may be determined from the curvature of the conduction band around the band minimum [1,2]. Despite of its simplicity, formula (1) can qualitatively account for the electromagnetic response of free carriers in a metal over a wide frequency range [3,4], including the low-frequency Hagen-Rubens regime $\omega<1 / \tau$, the infrared or optical regime $1 / \tau<\omega<\omega_{\mathrm{p}}$ called the relaxation regime, and the

transparent regime beyond the plasma frequency, $\omega>\omega_{\mathrm{p}} \equiv \sqrt{4 \pi n_{\mathrm{e}} e^{2} / m_{*}}$.

Experimentally observed spectra of $\tilde{\sigma}(\omega)$ for real metals [4,5] exhibit complicated structures that are not expected solely from (1). Optical conductivities of some alkali metals show an enhancement near $\hbar \omega \approx \varepsilon_{\mathrm{F}}$ (with $\varepsilon_{\mathrm{F}}$ being the Fermi energy), indicating an onset of interband transition from the lowest to higher conduction band [1-5]. In vacuum ultraviolet or x-ray regime such that $\omega>>\omega_{\mathrm{p}}$, excitation of a deep core electron to the conduction band gives rise to a sharp absorption edge [4,5]. Nevertheless, the low-frequency behavior of $\tilde{\sigma}(\omega)$ for $\hbar \omega<\varepsilon_{\mathrm{F}}$, where complicated interband transitions do not occur, has often been 
analyzed with the aid of the Drude formula [4].

Although the Drude theory is of historical importance, it has still been a target of renewed interest in the context of modern condensed matter physics. Recent development of computer simulation methods based on ab initio molecular dynamics has enabled one to calculate electric conductivities of real metals over a wide range of temperatures and densities and to compare them directly with the Drude formula [6]. Recent experiments observed Drudelike conductivities even in those exotic materials as two-dimensional Dirac Fermions in graphene [7] and a strongly correlated heavy-fermion metal $\mathrm{UPd}_{2} \mathrm{Al}_{3}$ [8]. An overview of modern electron transport theories for various types of condensed matter, including generalizations of the Drude formula, was given by Allen [9]. More [10] discussed the connection between the Drude formula and radiation theory in hot dense plasmas by emphasizing their quantum-mechanical aspects.

\section{CLASSICAL, SEMICLASSICAL, AND QUANTUM TREATMENTS}

Formula (1) can be obtained easily [1] through the classical equation of motion for a single electron with momentum $\mathbf{p}$, that is, $d \mathbf{p}(t) / d t=-e \mathbf{E}(t)-\mathbf{p}(t) / \tau$. This equation can be solved by setting $\mathbf{p}(t)=\mathbf{p}_{0} \exp (-i \omega t)$. The electric current density can then be calculated as $\mathbf{J}_{0}=-n_{\mathrm{e}} e \mathbf{p}_{0} / m_{*}$, arriving at Eq. (1). Here, $\tau$ is regarded merely as a phenomenological damping constant.

An electron is a quantum-mechanical particle exhibiting a wave-like character. In the semiclassical treatment $[1,2]$, each electron is expressed by a wave packet whose trajectory is described by a classical equation of motion for average position $\mathbf{r}$ and velocity $\hbar \mathbf{k} / m_{*}$, where $\mathbf{k}$ denotes the wave vector . In discussing the electric conduction, it is convenient to introduce a nonequilibrium distribution function $F(\mathbf{r}, \mathbf{k}, t)$ in the semiclassical phase space $\{\mathbf{r}, \mathbf{k}\}$, whose time evolution obeys the transport equation [1-3,11,12],

$$
\frac{\partial F(\mathbf{r}, \mathbf{k}, t)}{\partial t}+\frac{\hbar \mathbf{k}}{m_{*}} \cdot \frac{\partial F(\mathbf{r}, \mathbf{k}, t)}{\partial \mathbf{r}}-\frac{e \mathbf{E}(t)}{\hbar} \cdot \frac{\partial F(\mathbf{r}, \mathbf{k}, t)}{\partial \mathbf{k}}=-\frac{F(\mathbf{r}, \mathbf{k}, t)-f_{\mathbf{k}}^{(0)}}{\tau(\mathbf{k})} .
$$

Here, the relaxation-time approximation (RTA) [1-3,11,12] has been adopted on the right-hand 
side, where $\tau(\mathbf{k})$ represents the $k$-dependent collisional relaxation time. In the absence of the electric field, $F(\mathbf{r}, \mathbf{k}, t)$ coincides with the Fermi-Dirac distribution $f_{\mathbf{k}}^{(0)}$. For a freeelectron gas at zero temperature, $f_{\mathbf{k}}^{(0)}$ is given by a step function: $f_{\mathbf{k}}^{(0)}=1$ for $k \leq k_{\mathrm{F}}$, and $f_{\mathbf{k}}^{(0)}=0$ for $k>k_{\mathrm{F}}$, with $k_{\mathrm{F}}=\left(3 \pi^{2} n_{\mathrm{e}}\right)^{1 / 3}$ denoting the Fermi wave vector [1,2]. In the presence of a weak electric field $\mathbf{E}(t)=\mathbf{E}_{0} \exp (-i \omega t)$, a perturbative solution to equation (2) can be obtained in the form $F(\mathbf{r}, \mathbf{k}, t)=f_{k}^{(0)}+F_{1}(\mathbf{k}) \exp (-i \omega t)$. By evaluating the current density in accordance with $\mathbf{J}_{0}=-(2 e / \Omega) \sum_{\mathbf{k}}\left(\hbar \mathbf{k} / m_{*}\right) F_{1}(\mathbf{k})$, where $\Omega$ denotes the volume of the system, we recover Eq. (1) with $\tau$ being replaced by $\tau\left(k_{\mathrm{F}}\right)$ [1-3]. Thus, the fact that the electrons obey the quantum Fermi-Dirac distribution rather than the classical Boltzmann distribution affects the assessment of $\tau$ but does not alter the expression (1) itself. Microscopic expressions of $\tau(\mathbf{k})$ for various scattering mechanisms, such as acoustic/optical phonons and impurities, have been presented for semiconductors [11,12].

Validity of the RTA expression on the right-hand side of equation (2) was critically argued in [12], where it has been concluded that RTA can be justified if the scattering is either elastic or isotropic. In the present work, we shall treat nearly elastic scattering, such as the scattering by long-wavelength acoustic phonons, for which RTA is valid. An exception such as the scattering by optical phonons in polar materials will not be considered in this work.

In the quantum treatment, the dynamics of a many-electron system is governed by the time-dependent Schrödinger equation. In the initial unperturbed state, the electrons occupy a set of single-particle states $\left\{\left|\psi_{k}\right\rangle\right\}$ that diagonalize the single-particle Hamiltonian [13]. When a time-dependent electric field (here assumed to be parallel to z-axis) is applied, each electron undergoes a transition between different quantum states $\left|\psi_{k}\right\rangle$ and $\left|\psi_{k^{\prime}}\right\rangle$. The macroscopic electric conduction, which is essentially a collective phenomenon, is determined by the way how these single-particle states are populated in the presence of the electric field. Consequently, the real part of the conductivity can be expressed $[3,5,9,14,15]$ in terms of a square of the matrix elements of dipole transitions summed over all possible pair of states $k$ and $k^{\prime}$, namely,

$$
\operatorname{Re} \tilde{\sigma}(\omega)=\frac{2 \pi e^{2}}{\hbar \Omega m_{\mathrm{e}}{ }^{2} \omega} \sum_{k k^{\prime}}\left|\left\langle\psi_{k^{\prime}}\left|\frac{\hbar}{i} \frac{\partial}{\partial z}\right| \psi_{k}\right\rangle\right|^{2} \delta\left(\omega-\omega_{k^{\prime} k}\right)\left(f_{k}^{(0)}-f_{k^{\prime}}^{(0)}\right) \text {, }
$$


where $\hbar \omega_{k^{\prime} k} \equiv \varepsilon_{k^{\prime}}-\varepsilon_{k}$ corresponds to the energy difference between the two states. Equation (3) is generally referred to as the Kubo-Greenwood formula [3,5,6,9,14], which can be derived from the Kubo formula of general transport coefficients [9,15]. When the scattering is so weak that the mean-free path is longer than the interatomic spacing, the Greenwood-Kubo formula reduces to the Boltzmann transport theory [14]. Note that $\operatorname{Im} \tilde{\sigma}(\omega)$ can be obtained from $\operatorname{Re} \tilde{\sigma}(\omega)$ through the Kramers-Kronig relations [3,5].

The connection between equations (1) and (3) has been investigated through different approaches for different systems [3,5,14,16,17]. For a system of electrons in the presence of randomly distributed weak scatterers, Edwards [16] recast equation (3) into the Green's function formalism, and reproduced formula (1) at $\omega=0$ by using diagrammatic techinques. A simpler derivation of $\sigma(0)$ that avoids the use of diagrams can be found in the textbook of Mott and Davis [14], but the derivation for finite $\omega$ was suggested only in a heuristic way. The calculation of $\operatorname{Re} \tilde{\sigma}(\omega)$ for finite $\omega$ was carried out by Faber [17] for a liquid metal: He expressed the electron eigenfunction $\psi_{\mathbf{k}}$ as a wave group (superposition of plane waves with different wave vectors), and evaluated the matrix element of electron-ion scattering by summing up relevant intermediate processes represented graphically as ‘closed collapsed rings'. The derivation is lengthy, but his final result coincides with the real part of equation (1).

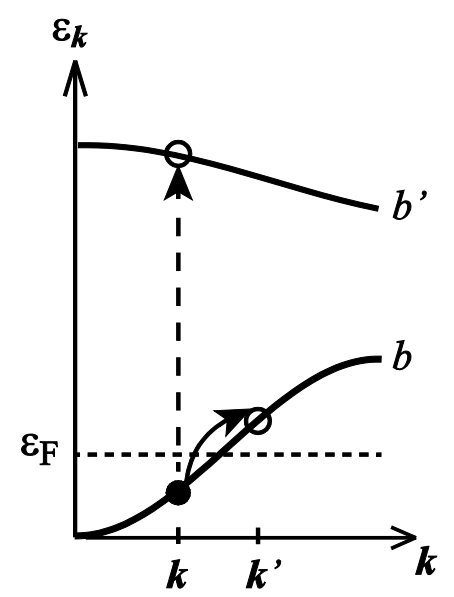

Figure 1. Interband (dashed arrow) and intraband (solid arrow) transitions across the Fermi level $\varepsilon_{\mathrm{F}}$.

Let us turn our attention to crystalline solids. The index $k$ in equation (3) can now be 
interpreted as the Bloch wave vector $\mathbf{k}$ and the band index $b$. When $\hbar \omega$ is larger than the energy gap, the conductivity is dominated by interband transitions $\{\mathbf{k} b\} \rightarrow\left\{\mathbf{k} b^{\prime}\right\}$, which manifests a vertical transition accompanying an absorption of a photon with frequency $\omega$ (see figure 1). The corresponding conductivity can be calculated [5] directly through equation (3). Instead, the process relevant to the Drude conductivity is an intraband transition $\{\mathbf{k} b\} \rightarrow\left\{\mathbf{k}^{\prime} b\right\}$, which may be regarded as a combined process of photoabsorption that accompanies a change in energy by $\hbar \omega$ and an absorption of a phonon that accompanies a change in the momentum $\mathbf{k} \rightarrow \mathbf{k}^{\prime}$ [5]. In this case, the matrix element in equation (3) must include the contribution of electron-phonon interaction. Bassani and Altarelli [5] indicated that, when this matrix element is evaluated approximately within the second-order perturbation theory, one obtains $\operatorname{Re} \tilde{\sigma}(\omega)=n_{\mathrm{e}} e^{2} / m_{*} \omega^{2} \tau \quad$ [equation (206) of Ref 5], which turns out equivalent to the highfrequency version $(\omega \tau>>1)$ of formula (1); the low-frequency behavior $(0<\omega \tau<1)$ remains unclarified.

Dressel and Grüner [3] attempted to derive formula (1) directly from the Kubo formula. Their derivation indicated in section 5.1.3, however, makes use of apparently wrong relations such as $\hbar \omega=\hbar^{2} k^{2} / 2 m_{\mathrm{e}}$ and $\hbar \omega=\hbar \omega_{s^{\prime} s}$; the latter equation is satisfied only for special combinations of $s$ and $s$ ' for a given $\omega$. The summation over $s$ and s', necessary to obtain the conductivity, is absent in some equations [e.g., their equation (5.1.33)] or inserted in wrong places [equation (5.1.32)], and it is not clear how this summation was finally evaluated. In addition, the relaxation time $\tau$ was introduced in an ad hoc manner without microscopic considerations of phonon-assisted processes mentioned above. Their derivation of the Drude formula cannot be justified.

To sum up, a quantum-mechanical derivation of the conductivity (1) for finite $\omega$ (especially for $0<\omega \tau<1$ ), that takes proper account of phonon-assisted intraband transitions, has not been clearly documented in standard textbooks. We shall show in the next section that the quantum kinetic equations based on the density-matrix formalism can reproduce formula (1) in a straightforward fashion.

\section{QUANTUM KINETIC EQUATIONS}


The density-matrix formulations of quantum electron dynamics put forth by Kohn and Luttinger [18] and developed by the author [19] provide the basis for the following analysis. Let us consider a single-band metal with one-particle energies $\left\{\varepsilon_{\mathbf{k}}\right\}$ and wave functions $\left\{\psi_{\mathbf{k}}(\mathbf{r})\right\}$. When a weak electric field $\mathbf{E}(t)$ is applied, the total Hamiltonian may be written as [18]

$$
H(t)=\sum_{\mathbf{k} \sigma} \varepsilon_{\mathbf{k}} C_{\mathbf{k} \sigma}^{\dagger} C_{\mathbf{k} \sigma}+\sum_{\mathbf{k} \mathbf{k}^{\prime} \sigma}\left[-\mathbf{d}_{\mathbf{k} \mathbf{k}^{\prime}} \cdot \mathbf{E}(t)+V_{\mathbf{k} \mathbf{k}^{\prime}}\right] C_{\mathbf{k} \sigma}^{\dagger} C_{\mathbf{k}^{\prime} \sigma} \cdot
$$

Here, $c_{\mathbf{k} \sigma}^{\dagger}$ and $c_{\mathbf{k} \sigma}$ are the creation and annihilation operators [20], respectively, for a state with wave vector $\mathbf{k}$ and spin $\sigma$. The summation over $\mathbf{k}$ and $\mathbf{k}$ ' in the last term should omit the contribution from $\mathbf{k}=\mathbf{k}^{\prime}$. The interaction of an electron with the electric field is treated with the dipole matrix element $\quad \mathbf{d}_{\mathbf{k k}^{\prime}} \equiv\left\langle\psi_{\mathbf{k}}|-e \mathbf{r}| \psi_{\mathbf{k}^{\prime}}\right\rangle$. The quantity $\quad V_{\mathbf{k k}^{\prime}} \equiv\left\langle\psi_{\mathbf{k}}|V| \psi_{\mathbf{k}^{\prime}}\right\rangle$ represents the matrix element of the electron-phonon scattering: Since the characteristic phonon energy is much smaller than the Fermi energy, the scattering may be regarded as nearly elastic [5]. We also note that the scattering is anisotropic in the sense that $V_{\mathbf{k k}^{\prime}}$ depends generally on $\mathbf{k}-\mathbf{k}^{\prime}$.

We now examine the time evolution of this system. We introduce a one-particle density matrix through the definition $\left\langle\rho_{\mathbf{k} \mathbf{k}^{\prime} \sigma}(t)\right\rangle \equiv\left\langle\Psi(t)\left|c_{\mathbf{k} \sigma}^{\dagger} c_{\mathbf{k}^{\prime} \sigma}\right| \Psi(t)\right\rangle$, where $\Psi(t)$ denotes the wave function of the total system. By taking the time derivative of both sides of this equation and making use of the relation $i \hbar \partial \Psi(t) / \partial t=H(t) \Psi(t)$ (i.e., the time-dependent Schrödinger equation), we obtain the Heisenberg equation of motion $[18,19]$

$$
i \hbar \frac{\partial\left\langle\rho_{\mathbf{k k}^{\prime} \sigma}(t)\right\rangle}{\partial t}=\left\langle\Psi(t)\left|\left[c_{\mathbf{k} \sigma}^{\dagger} c_{\mathbf{k}^{\prime} \sigma}, H(t)\right]\right| \Psi(t)\right\rangle,
$$

where the square brackets denote the commutator, $[A, B] \equiv A B-B A$. The right-hand side of (5) can be evaluated with the aid of equation (4) and the Fermion anticommutation relation [20] $\left\{c_{\mathbf{k} \sigma}^{\dagger}, c_{\mathbf{k}^{\prime} \sigma^{\prime}}\right\} \equiv c_{\mathbf{k} \sigma}^{\dagger} C_{\mathbf{k}^{\prime} \sigma^{\prime}}+c_{\mathbf{k}^{\prime} \sigma^{\prime}} c_{\mathbf{k} \sigma}^{\dagger}=\delta_{\mathbf{k} \mathbf{k}^{\prime}} \delta_{\sigma \sigma^{\prime}}$ to yield the quantum kinetic equation $[18,19]$

$$
\frac{\partial\left\langle\rho_{\mathbf{k k}^{\prime}}(t)\right\rangle}{\partial t}=i \omega_{\mathbf{k k}^{\prime}}\left\langle\rho_{\mathbf{k k}^{\prime}}(t)\right\rangle+\frac{i}{\hbar} \sum_{\mathbf{q}}\left[\mathbf{d}_{\mathbf{k}^{\prime} \mathbf{q}} \cdot \mathbf{E}(t)\left\langle\rho_{\mathbf{k q}}(t)\right\rangle-\mathbf{d}_{\mathbf{q} \mathbf{k}} \cdot \mathbf{E}(t)\left\langle\rho_{\mathbf{q k}^{\prime}}(t)\right\rangle\right]
$$




$$
-\frac{i}{\hbar} \sum_{\mathbf{q}}\left[V_{\mathbf{k}^{\prime} \mathbf{q}}\left\langle\rho_{\mathbf{k q}}(t)\right\rangle-V_{\mathbf{q k}}\left\langle\rho_{\mathbf{q k}^{\prime}}(t)\right\rangle\right]
$$

The density matrix is independent of the spin in the problem under consideration and hence the index $\sigma$ will be omitted hereafter.

To proceed further, we assume the free-electron approximation, $\psi_{\mathbf{k}}(\mathbf{r})=(1 / \sqrt{\Omega}) e^{i \mathbf{k} \cdot \mathbf{r}}$. The dipole matrix element can then be calculated as

$$
\mathbf{d}_{\mathbf{k} \mathbf{k}^{\prime}}=\frac{1}{\Omega} \int d \mathbf{r} e^{-i \mathbf{k} \cdot \mathbf{r}}(-e) \mathbf{r} e^{i \mathbf{k}^{\prime} \cdot \mathbf{r}}=\frac{-e}{i \Omega} \int d \mathbf{r} \frac{\partial}{\partial \mathbf{k}^{\prime}} e^{-i\left(\mathbf{k}-\mathbf{k}^{\prime}\right) \cdot \mathbf{r}}=i e \frac{(2 \pi)^{3}}{\Omega} \frac{\partial \delta\left(\mathbf{k}-\mathbf{k}^{\prime}\right)}{\partial \mathbf{k}^{\prime}} .
$$

It is convenient to separate out the diagonal component $f_{\mathbf{k}}(t) \equiv\left\langle\rho_{\mathbf{k} \mathbf{k}}(t)\right\rangle$ which represents the average occupation number of state $\mathbf{k}[18,19]$. Equation (6) is thus split into two parts,

$$
\begin{gathered}
\frac{\partial f_{\mathbf{k}}(t)}{\partial t}=\frac{e \mathbf{E}(t)}{\hbar} \cdot \frac{\partial f_{\mathbf{k}}(t)}{\partial \mathbf{k}}-\frac{i}{\hbar} \sum_{\mathbf{q}}\left[V_{\mathbf{k}, \mathbf{k}+\mathbf{q}}\left\langle\rho_{\mathbf{k}, \mathbf{k}+\mathbf{q}}(t)\right\rangle-V_{\mathbf{k}+\mathbf{q}, \mathbf{k}}\left\langle\rho_{\mathbf{k}+\mathbf{q}, \mathbf{k}}(t)\right\rangle\right], \\
\frac{\partial\left\langle\rho_{\mathbf{k}, \mathbf{k}+\mathbf{q}}(t)\right\rangle}{\partial t}=i \omega_{\mathbf{k}, \mathbf{k}+\mathbf{q}}\left\langle\rho_{\mathbf{k}, \mathbf{k}+\mathbf{q}}(t)\right\rangle+\frac{e \mathbf{E}(t)}{\hbar} \cdot \frac{\partial\left\langle\rho_{\mathbf{k}, \mathbf{k}+\mathbf{q}}(t)\right\rangle}{\partial \mathbf{k}}-\frac{i}{\hbar} V_{\mathbf{k}+\mathbf{q}, \mathbf{k}}\left[f_{\mathbf{k}}(t)-f_{\mathbf{k}+\mathbf{q}}(t)\right] \\
-\frac{i}{\hbar} \sum_{\mathbf{q}^{\prime} \neq 0, \mathbf{q}}\left[V_{\mathbf{k}+\mathbf{q}, \mathbf{k}+\mathbf{q}^{\prime}}\left\langle\rho_{\mathbf{k}, \mathbf{k}+\mathbf{q}^{\prime}}(t)\right\rangle-V_{\mathbf{k}+\mathbf{q}^{\prime} \mathbf{k}}\left\langle\rho_{\mathbf{k}+\mathbf{q}^{\prime}, \mathbf{k}+\mathbf{q}}(t)\right\rangle\right] \quad(\mathbf{q} \neq 0) .
\end{gathered}
$$

It is important to note here that the k-derivative terms in equations (8a) and (8b) have stemmed from relation (7). We have previously shown [19] that the last term on the right-hand side of equation (8b), representing the nonlinear effect, plays a role only for a strong perturbation, and hence it can be neglected if the scattering is weak. Even if the scattering is sufficiently strong, we have confirmed through several numerical examples [19] that the condition $0 \leq f_{\mathbf{k}}(t) \leq 1$ is properly satisfied if the nonlinear term in (8b) is retained. Also, it follows from (8a) that $\sum_{\mathbf{k}} \partial f_{\mathbf{k}}(t) / \partial t=0$, which ensures that the total number of electrons (or the trace of the density matrix) is conserved.

In the presence of an oscillating electric field $\mathbf{E}(t)=\mathbf{E}_{0} \exp (-i \omega t+\Gamma t)$, where $\Gamma$ is a positive infinitesimal assuring adiabatic turn-on of the field, we seek for the solutions to equations (8a) and (8b) in the form 


$$
f_{\mathbf{k}}(t)=f_{\mathbf{k}}^{(0)}+f_{\mathbf{k}}^{(1)} \exp (-i \omega t+\Gamma t),\left\langle\rho_{\mathbf{k}, \mathbf{k}+\mathbf{q}}(t)\right\rangle=\left\langle\rho_{\mathbf{k}, \mathbf{k}+\mathbf{q}}^{(1)}\right\rangle \exp (-i \omega t+\Gamma t) .
$$

Here, the superscript ' $(0)$ ' refers to a quantity in the unperturbed state $(\mathbf{E}=0)$, while '(1)' corresponds to a first-order quantity induced by $\mathbf{E}(t)$. The first-order solution to equation (8b) is then obtained as

$$
\left\langle\rho_{\mathbf{k}, \mathbf{k}+\mathbf{q}}^{(1)}\right\rangle=\frac{i V_{\mathbf{k}+\mathbf{q}, \mathbf{k}}\left[f_{\mathbf{k}}^{(1)}-f_{\mathbf{k}+\mathbf{q}}^{(1)}\right]}{\hbar\left[i\left(\omega-\omega_{\mathbf{k}+\mathbf{q}, \mathbf{k}}\right)-\Gamma\right]}, \quad\left\langle\rho_{\mathbf{k}+\mathbf{q}, \mathbf{k}}^{(1)}\right\rangle=\left\langle\rho_{\mathbf{k}, \mathbf{k}+\mathbf{q}}^{(1)}\right\rangle^{*},
$$

which in turn is substituted into equation (8a) to yield

$$
(-i \omega+\Gamma) f_{\mathbf{k}}^{(1)}=\frac{e \mathbf{E}_{0}}{\hbar} \cdot \frac{\partial f_{\mathbf{k}}^{(0)}}{\partial \mathbf{k}}-\frac{2}{\hbar^{2}} \sum_{\mathbf{k}^{\prime}}\left|V_{\mathbf{k} \mathbf{k}}\right|^{2}\left[f_{\mathbf{k}}^{(1)}-f_{\mathbf{k}^{\prime}}^{(1)}\right] \frac{\Gamma}{\left(\omega-\omega_{\mathbf{k} \mathbf{k}}\right)^{2}+\Gamma^{2}} .
$$

Here, one observes a similarity between equations (2) and (11): The first term on the right-hand side of equation (11) represents the quiver motion of an electron in an oscillating field, whereas the second term provides a microscopic expression of the electron-phonon scattering corresponding to the relaxation term on the right-hand side of equation (2).

The remaining task is to solve equation (11) for $f_{\mathbf{k}}^{(1)}$; this procedure is similar to the semiclassical case. In so doing, the anisotropy of $f_{\mathbf{k}}^{(1)}$ can be analyzed with the Legendrepolynomial expansion method [11]. Since the anisotropy is dominated by a deformation of the Fermi sphere along the direction of $\mathbf{E}_{0}$, we may retain the expansion only up to first order as [11]

$$
f_{\mathbf{k}}^{(1)} \approx f_{k}^{(1,0)}+f_{k}^{(1,1)} \cos \theta,
$$

where $\theta$ is the angle between $\mathbf{k}$ and $\mathbf{E}_{0}$. Note that $f_{k}^{(1,0)}$ and $f_{k}^{(1,1)}$ depend only on $k \equiv|\mathbf{k}|$.

In order to perform the $\mathbf{k}^{\prime}$-summation in equation (11), it is convenient to set up a coordinate system displayed in figure 2, in which $\mathbf{k}=(0,0, k), \mathbf{E}_{0}=\left(E_{0} \sin \theta, 0, E_{0} \cos \theta\right)$, and $\mathbf{k}^{\prime}=\left(k^{\prime} \sin \theta_{k^{\prime} k} \cos \varphi^{\prime}, k^{\prime} \sin \theta_{k^{\prime} k} \sin \varphi^{\prime}, k^{\prime} \cos \theta_{k^{\prime} k}\right)$. The angle $\theta^{\prime}$ between $\mathbf{k}^{\prime}$ and $\mathbf{E}_{0}$ satisfies the relation $\cos \theta^{\prime}=\sin \theta_{k^{\prime} k} \sin \theta \cos \varphi^{\prime}+\cos \theta_{k^{\prime} k} \cos \theta$. The unperturbed Fermi surface is assumed to be spherical; it then follows that the Fermi distribution $f_{\mathbf{k}}^{(0)}$ can be written simply as $f_{k}^{(0)}$, and that $V_{\mathbf{k} \mathbf{k}}$ is independent of $\varphi^{\prime}$. By substituting equation (12) into (11) and performing the angular integration $\int_{-1}^{1} d(\cos \theta)(\cdots)$, we find a trivial solution 
$f_{k}^{(1,0)}=0$ for the zeroth-order term. The solution to the first-order term can likewise be obtained through operating the integration $\int_{-1}^{1} d(\cos \theta) \cos \theta(\cdots)$ to both sides of equation (11); the result is

$$
(-i \omega+\Gamma) f_{k}^{(1,1)}=\frac{e E_{0}}{\hbar} \frac{\partial f_{k}^{(0)}}{\partial k}-\frac{2}{\hbar^{2}} \sum_{\mathbf{k}^{\prime}}\left|V_{\mathbf{k}^{\prime} \mathbf{k}}\right|^{2}\left[f_{k}^{(1,1)}-f_{k^{\prime}}^{(1,1)} \cos \theta_{k^{\prime} \mathbf{k}}\right] \frac{\Gamma}{\left(\omega-\omega_{\mathbf{k} \mathbf{k}}\right)^{2}+\Gamma^{2}} .
$$

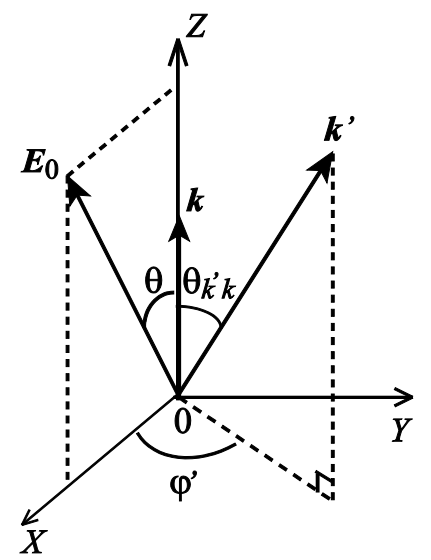

Figure 2. Geometry of the three vectors $\mathbf{k}, \mathbf{k}^{\prime}$, and $\mathbf{E}_{0}$.

Since $\hbar \omega<<\varepsilon_{\mathrm{F}}=\hbar^{2} k_{\mathrm{F}}^{2} / 2 m_{*}$ for intraband transitions, we may set $\omega \approx 0$ in the last term on the right-hand side of equation (13). Also, as we shall see below in equation (14), the dominant contribution to $f_{k}^{(1,1)}$ arises from $k \approx k^{\prime} \approx k_{\mathrm{F}}$ and hence we may set $f_{k^{\prime}}^{(1,1)} \approx f_{k}^{(1,1)}$. As a consequence, the solution to equation (13) can be expressed in the limit of $\Gamma \rightarrow 0$ as

$$
f_{k}^{(1,1)}=\frac{\tau(k)}{1-i \omega \tau(k)} \frac{e E_{0}}{\hbar} \frac{\partial f_{k}^{(0)}}{\partial k} \approx-\frac{\tau(k)}{1-i \omega \tau(k)} \frac{e E_{0}}{\hbar} \delta\left(k-k_{\mathrm{F}}\right),
$$

where the last equality is valid at zero temperature; $\tau(k)$ accounts for the usual $k$-dependent relaxation time of electron-phonon scattering [1,2,5,12]

$$
\frac{1}{\tau(k)}=\frac{2 \pi}{\hbar} \sum_{\mathbf{k}^{\prime}}\left|V_{\mathbf{k}^{\prime} \mathbf{k}}\right|^{2}\left(1-\cos \theta_{k^{\prime} \mathbf{k}}\right) \delta\left(\varepsilon_{\mathbf{k}^{\prime}}-\varepsilon_{\mathbf{k}}\right) \text {. }
$$

Here, the delta function, which originates from the Lorentzian in the last term of equation (13), manifests the energy conservation in an elastic scattering. The induced electric current density 
along the direction of $\mathbf{E}_{0}$ can be calculated with the aid of equations (12) and (14) as

$$
J_{0}=-\frac{2 e}{\Omega} \sum_{\mathbf{k}} \frac{\hbar k \cos \theta}{m_{*}} f_{\mathbf{k}}^{(1)}=\frac{n_{\mathrm{e}} e^{2} E_{0}}{m_{*}} \frac{\tau\left(k_{\mathrm{F}}\right)}{1-i \omega \tau\left(k_{\mathrm{F}}\right)} .
$$

Thus, we finally obtain the complex conductivity

$$
\tilde{\sigma}(\omega)=\frac{J_{0}}{E_{0}}=\frac{n_{\mathrm{e}} e^{2} \tau\left(k_{\mathrm{F}}\right)}{m_{*}} \frac{1}{1-i \omega \tau\left(k_{\mathrm{F}}\right)},
$$

which turns out to coincide exactly with the Drude formula (1).

While the earlier derivation by Bassani and Altarelli [5] was limited to high-frequency regime $\omega \tau>1$ because of the assumption $\left|V_{\mathbf{k} \mathbf{k}}\right|<<\hbar \omega$, the present theory does not make such an assumption, leading to a successful derivation of formula (17) applicable to any $\omega$.

\section{CONCLUDING REMARKS}

We have demonstrated a quantum-mechanical proof of the Drude formula (1) through solutions to the quantum kinetic equations that are based on the density-matrix formulation of the timedependent Schrödinger equation. Throughout our derivation, we have merely assumed that (i) the electrons are mutually noninteracting, (ii) the unperturbed Fermi surface is isotropic, and (iii) the electron-phonon scattering is quasielastic and treated linearly. In particular, validity of formula (1) for the frequency range $0<\omega \tau<1$, which could not be indicated easily in previous works based on the Kubo-Greenwood formula, has been proven successfully in a straightforward manner without mathematical complexities. It is essential to treat the intraband dipole matrix element (7) and the matrix element of quasielastic electron-phonon scattering on an equal footing, as manifested in (4) and (11).

The Kubo-Greenwood formula may be most useful for analyzing interband transitions, whereas its application to phonon-assisted intraband transition needs a careful analysis [3,5]. In contrast, the semiclassical Boltzmann equation is suitable for intraband transport but incapable of treating interband transitions [1]. A salient feature of the density-matrix equation presented in this paper is the rigorousness supported by the quantum theory as well as its similarity with the familiar Boltzmann transport equation; it provides physical insight into the 
link between the microscopic Schrödinger equation and macroscopic transport.

The density-matrix theory offers a general framework of quantum dynamics so that it would be potentially applicable to various subjects of condensed matter physics. Recent density-matrix studies of stochastic dynamics in open quantum systems and quantum optics can be found in Ref [21].

\section{ACKNOWLEDGMENTS}

This work was supported in part through Grant-in-Aid for Scientific Research (A) provided by JSPS. 


\section{References}

[1] N.W. Ashcroft and N.D. Mermin 1976 Solid State Physics (CA: Brooks/Cole) chapters 1, 2, 12 and 13

[2] Ziman J M 1992 Principles of the Theory of Solids 2nd edn (Cambridge: Cambridge University Press) chapter 7

[3] Dressel M and Grüner G 2002 Electrodynamics of Solids (Cambridge: Cambridge University Press) section 5.1

[4] Lynch D W and Hunter W R 1998 An Introduction to the Data for Several Metals: in Handbook of Optical Constants of Solids II, edited by E.D. Parik (CA: Academic Press) p 341

[5] Bassani F and Altarelli M 1983 Interaction of radiation with condensed matter: in Handbook of Synchrotron Radiation Vol. 1 edited by E E Koch (North-Holland) p 463

[6] Desjarlais M P, Kress J D and Collins L A 2002 Electrical conductivity for warm, dense aluminum plasmas and liquids Phys. Rev. E 66 025401(R)

[7] Horng J, Chen C -F, Geng B, Girit C, Zhang Y, Hao Z, Bechtel H A, Martin M, Zettl A, Crommie M F, Shen Y R and Wang F 2011 Drude conductivity of Dirac fermions in graphene Phys. Rev. B 83165113

[8] Scheffler M, Dressel M, Jourdan M and Adrian H 2005 Extremely slow Drude relaxation of correlated electrons Nature 4381135

[9] Allen P B 2006 Conceptual Foundations of Materials: A Standard Model for Ground- and Excited-State Properties: Contemporary Concepts of Condensed Matter Science, edited by S G Louie and M L Cohen (Elsevier) p 165

[10] More R M 2000 Quantum Conduction Phenomena in Hot Dense Plasmas J. Plasma \& Fusion Res. 76623

[11] Ridley B K 2013 Quantum Processes in Semiconductors 5th edn (Oxford: Oxford University Press) chapters 11 and 12

[12] Lundstrom M 2000 Fundamentals of Carrier Transport 2nd edn (Cambridge: Cambridge University Press) chapters 2 and 3

[13] Fulde P 1995 Electron Correlations in Molecules and Solids (Springer) chapters 2 and 3 
[14] Mott N F and Davis E A 1971 Electronic Processes in Non-Crystalline Materials (Oxford: Clarendon Press) section 2.3

[15] Kubo R 1957 Statistical-Mechanical Theory of Irreversible Processes I. General Theory and Simple Applications to Magnetic and Conduction Problems J. Phys. Soc. Japan 12570

[16] Edwards S F 1958 A New Method for the Evaluation of Electric Conductivity Philos. Mag. 31020

[17] Faber T E 1972 An Introduction to the Theory of Liquid Metals (NY: Cambridge University Press) sections 5.14 and 5.29

[18] Kohn W and Luttinger J M 1957 Quantum theory of electrical transport phenomena Phys. Rev. 108590

[19] Kitamura H 2014 Density-matrix theory of quantum dynamics under a strong external field switched on nonadiabatically Int. J. Quant. Chem. 1141518

[20] Fetter A L and Walecka J D 2003 Quantum Theory of Many-Particle Systems (NY: Dover) chapter 1

[21] Breuer H -P and Petruccione F 2006 The Theory of Open Quantum Systems (NY: Oxford University Press) 